\title{
Radiation Exposure from CT Examinations in Japan
}

\author{
Yoshito Tsushima ${ }^{*}{ }^{\dagger}$, Ayako Taketomi-Takahashi ${ }^{\dagger}$, Hiroyuki Takei ${ }^{\dagger}$, Hidenori Otake ${ }^{\dagger}$, Keigo Endo ${ }^{\dagger}$
}

\begin{abstract}
Background: Computed tomography (CT) is the largest source of medical radiation exposure to the general population, and is considered a potential source of increased cancer risk. The aim of this study was to assess the current situation of $\mathrm{CT}$ use in Japan, and to investigate variations in radiation exposure in $\mathrm{CT}$ studies among institutions and scanners.

Methods: Data-sheets were sent to all 126 hospitals and randomly selected 14 (15\%) of 94 clinics in Gunma prefecture which had CT scanner(s). Data for patients undergoing CT during a single month (June 2008) were obtained, along with CT scan protocols for each institution surveyed. Age and sex specific patterns of CT examination, the variation in radiation exposure from $\mathrm{CT}$ examinations, and factors which were responsible for the variation in radiation exposure were determined.

Results: An estimated 235.4 patients per 1,000 population undergo CT examinations each year, and 50\% of the patients were scanned in two or more anatomical locations in one CT session. There was a large variation in effective dose among hospitals surveyed, particularly in lower abdominal CT (range, 2.6-19.0 mSv). CT examinations of the chest and upper abdomen contributed to approximately $73.2 \%$ of the collective dose from all CT examinations. It was estimated that in Japan, approximately 29.9 million patients undergo $C T$ annually, and the estimated annual collective effective dose in Japan was $277.4 * 10^{3} \mathrm{~Sv}$ person. The annual effective dose per capita for Japan was estimated to be $2.20 \mathrm{mSv}$.
\end{abstract}

Conclusions: There was a very large variation in radiation exposure from $\mathrm{CT}$ among institutions surveyed. CT examinations of the chest and upper abdomen were the predominant contributors to the collective dose.

\section{Background}

Since computed tomography (CT) was introduced to medical practice, this diagnostic X-ray technique has provided great benefits for health care. In most circumstances, the risk to an individual patient of developing a malignant tumour because of $\mathrm{CT}$ is low and acceptable compared to the substantial benefit, although there is a large uncertainty in risk estimates at these dose levels. However, the large number of people exposed means that even small individual risks could translate into a considerable number of cancer deaths [1,2]. In the UK (2005-2006), approximately $60 \%$ of the total radiology collective effective dose was from CT [3]. In Germany (2000-2005), the contribution of CT to the collective effective dose for cancer patients from all X-ray procedures was approximately $82 \%$ [4]. In the USA, about $67 \%$ of the collective effective dose in diagnostic radiology was due to CT scanning [5]. These

\footnotetext{
* Correspondence: tyoshito@showa.gunma-u.ac.jp

† Contributed equally

Department of Diagnostic Radiology and Nuclear Medicine, Gunma University Hospital 3-39-22 Showa-machi, Maebashi, Gunma, Japan
}

data indicated that CT represents more than half the radiation exposure from diagnostic imaging. In Japan, there is unfortunately no such reliable data regarding radiation exposure from radiological imaging, but the situation is likely not very different.

We surveyed all individual patients undergoing CT examinations during a period of one month in a single prefecture (state) in Japan. Age and sex distribution, anatomical locations scanned and radiation exposure of the patients receiving scans were noted.

\section{Methods}

We collected data concerning individual patients undergoing CT in Gunma prefecture during a single month (June 2008), along with the standard CT scan protocols of each scanner used in the respective institutions. For each individual patient, organ doses were calculated and the effective doses were obtained (using the standard $\mathrm{CT}$ scanning protocol of each institute). From these data, the current situation of CT examinations in Japan, with particular attention paid to radiation exposure, was 
estimated. The epidemiological research ethics committee of Gunma University Faculty of Medicine approved this survey, and did not require any informed consent from each patient, since all the data used were anonymous and the data collection did not affect patient management in any way.

\section{- Collecting the data of individual patients undergoing CT and standard $\mathrm{CT}$ scanning protocols}

A questionnaire was mailed to the chief radiologic technologist in all 126 hospitals and randomly selected 14 (15\%) of 94 clinics which had CT scanner(s) in Gunma prefecture (state). Gunma prefecture is located approximately $100 \mathrm{~km}$ north of Tokyo, and its population is two million. The age distribution in Gunma prefecture is very similar to that of Japan as a whole (Figure 1) [6], and we suspect that the medical environment in Gunma prefecture probably reflects that of the Japanese national average (Table 1) [7-11]. The chief technologist in each hospital was asked to make a list of all patients who underwent CT during a period of one month (June 2008). The data of each patient (CT session) consisted of the patient's age and sex, anatomical location of the CT scan and number of scans. When a patient returned for a second or more CT session on a different day, the sessions were counted as two different patients each undergoing a single CT session. The anatomical locations were divided into head, face, neck, chest, upper abdomen, lower abdomen (pelvis) and other.

In many patients, more than two anatomical locations were scanned, and sometimes more than two scans were performed on one anatomical location in a single CT session. Therefore, we defined the number of examinations as the number of anatomical locations scanned, and the number of scans as the total number of scans for each anatomical location. For example, if a patient underwent unenhanced CT of the upper abdomen and enhanced CT of the upper and lower abdomen in a single CT session, the number of CT examinations was two, and the number of scans of the upper abdomen was two and that of the lower abdomen was one.

From the data obtained, the mean numbers of the hospitals or clinics were calculated, and were multiplied by the number of CT scanners to estimate the total number of patients, examinations, and scans. These numbers multiplied by 12 were estimated as being the annual number of each in Gunma prefecture. The population of Japan is approximately 127 million and that of Gunma prefecture is two million, so these annual numbers for Gunma prefecture were multiplied by $127 / 2$ to estimate the annual numbers for those in Japan.

Inquiries were also made on the model of the CT scanner and standard scanning protocols for each anatomical location (tube voltage $[\mathrm{kV}]$, tube current $[\mathrm{mA}]$, rotation time [sec], collimation [mm], and pitch). Use of an automatic exposure control (AEC) system was also noted, if applicable.

\section{- Calculation of the organ doses for each patient}

We employed ImPACT CT Patient Dosimetry Calculator version $0.99 \mathrm{x}$ http://www.impactscan.org/. This software, which is constructed using a Microsoft Excel

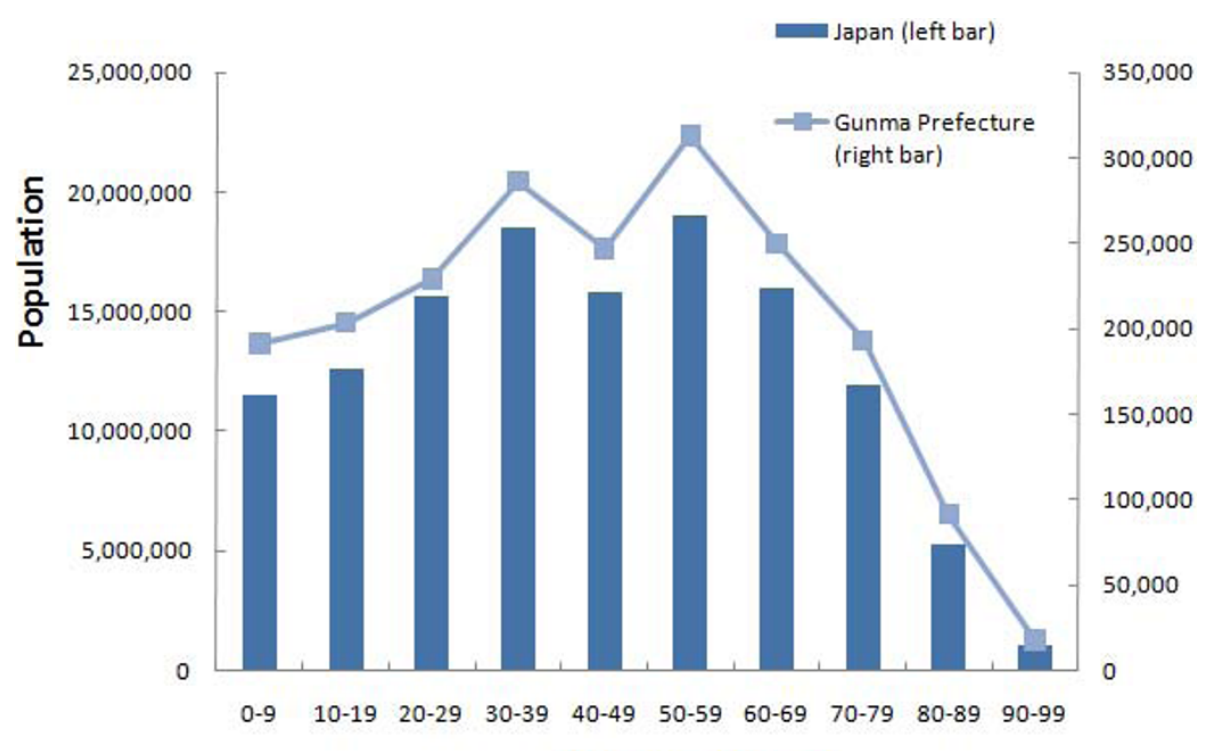

Age Range (y.o.)

Figure 1 Age distribution of the population of Japan and Gunma prefecture. The distributions are very similar. 
Table 1 Medical environment of Gunma prefecture

\begin{tabular}{llll}
\hline Variables & Gunma prefecture & National average & $\begin{array}{l}\text { Year of the data } \\
\text { (references) }\end{array}$ \\
\hline Population density $\left(\mathbf{k m}^{\mathbf{2}}\right)$ & 318 & 340 & 2006 \\
Annual income per capita (Japanese yen) & $2,828,000$ & $2,978,000$ & $2004(21)$ \\
Number of hospitals $(\mathbf{1 0 0 , 0 0 0}$ population) & 6.9 & 6.9 & $2007(19)$ \\
Number of beds $(\mathbf{1 0 0 , 0 0 0}$ population) & 1263 & 1268 & $2007(20)$ \\
Number of physicians (/100,000 population) & 208.6 & 217.5 & $2006(17)$ \\
Annual medical cost per capita (Japanese yen) & 343,000 & 386,000 & $2005(18)$ \\
\hline
\end{tabular}

The annual medical cost per population in Gunma prefecture was about $10 \%$ lower compared to the national average, but other variables are almost average.

spreadsheet, is a tool for calculating patient organ and effective doses from CT. It makes use of the NRPB Monte Carlo dose data sets produced in NRPB-SR250 [12], which provides normalized organ dose data for irradiation of a mathematical phantom by a range of $\mathrm{CT}$ scanners.

For each patient, organ doses $(\mathrm{mSv})$ were calculated using this software. The z-axis (axial) extent of the scan for each type of CT examination (anatomical location) were fixed as follows: $14 \mathrm{~cm}$ for head, $10 \mathrm{~cm}$ for face, $10.5 \mathrm{~cm}$ for neck, $25 \mathrm{~cm}$ of chest, $20 \mathrm{~cm}$ for upper abdomen, and $25 \mathrm{~cm}$ for lower abdomen. Data for most modern CT scanners of major vendors were included in this software. If data from a CT scanner used in a hospital or clinic in this study was not found in the data included in this software, available data from the most similar CT scanner was used.

The phantom used to produce the Monte Carlo data sets in NRPB-SR250 [12] is based on a mathematical representation of an average adult, but does not address the issue of dose to paediatric patients. Therefore, in this study, we calculated organ doses only for the patients older than 20 years of age.

\section{- Estimations of effective dose for one scan or examination and annual collective dose in Japan}

Estimated effective doses $(\mathrm{mSv})$ per scan or examination for each anatomical location were obtained by using the same software. From the values obtained, collective doses during the single month in Gunma prefecture were estimated, then, multiplied by 12 to estimate the annual collective doses. The annual collective doses in Japan attributable to $\mathrm{CT}$ were estimated by multiplying this value by $127 / 2$. The annual effective dose per capita was also estimated.

To find which factors may contribute to the estimated effective doses, these were correlated with hospital size (number of in-patient beds), the number of patients undergoing $\mathrm{CT}$ in one month, and the row number of the CT detector by using a linear regression analyses.

\section{Results}

- Data collection (Table 2)

Of 132 CT scanners in 126 hospitals, complete data were provided for $63 \mathrm{CT}$ scanners (48\%) of 57 hospitals. 61 hospitals reported only scanning protocols and the number of patients during the month surveyed, so scanning protocols and the number of the patients undergoing CT examinations for 124 of $132 \mathrm{CT}$ scanners (94\%) were obtained. Of 14 clinics (14 CT scanners) to which the questionnaire was sent, complete data were provided by six clinics.

Complete data for individual patients was collected for 19,013 patients scanned by 69 CT scanners.

\section{- Numbers of patients, CT examinations, and scanning}

The estimated annual number of adult patients who underwent CT in Gunma prefecture during the month surveyed was 471,557 (Table 2). The estimated annual number of patients undergoing CT per 1000 population would be 235.4 . In approximately $50 \%$ of the patients, more than two anatomical locations were examined in one CT session; the estimated annual number of examinations in Japan was 45.4 million (Figure 2; Table 3). In addition, approximately $37 \%$ of the patients underwent more than two scans of the same anatomical location (for instance, unenhanced and enhanced CT). CT examinations of the chest were the most frequent (27\%), closely followed by examinations of the upper abdomen (23\%), the neck (22\%) and head (20\%), with examinations of the lower abdomen (5\%) and face (3\%) being much less frequent. The number of CT examinations for men were larger than those for women.

From these data of Gunma prefecture, an estimated 29.9 million patients are scanned annually in Japan. The estimated annual number of scans in Japan was approximately 62.5 million (Figure 2). The annual number of pediatric patients undergoing CT in Japan was estimated as 1.43 million. The age and sex distribution for each anatomical location scanned were summarized in Tables 4, 5 . 
Table 2 Number of CT scanners in Gunma prefecture and data collection

\begin{tabular}{|c|c|c|c|c|c|c|}
\hline & \multicolumn{3}{|c|}{ Number of CT scanners } & \multicolumn{3}{|c|}{ Estimated annual number of patients } \\
\hline & \multirow[t]{2}{*}{ Total } & \multicolumn{2}{|r|}{ Data obtained (\%) } & \multirow[b]{2}{*}{ Mean } & \multirow[b]{2}{*}{ Range } & \multirow[b]{2}{*}{ Estimated total number } \\
\hline & & Complete data & Scanning protocol \& Number of patients* & & & \\
\hline Hospitals & 132 & 63 & 61 & 2,571 & $36-23,616$ & 339,393 \\
\hline Clinics & $94^{* *}$ & 6 & 0 & 1,406 & $288-2,664$ & 132,164 \\
\hline Total & 226 & 69 & 61 & & & 471,557 \\
\hline
\end{tabular}

*No individual patients' data were provided.

**Data requests sent to 14 randomly-selected clinics.

\section{- The estimated effective dose, and their distribution}

The mean effective doses from a single scan or examination for each anatomical location were summarized in Table 6 along with comparable data reported from European countries [8-11], and the distributions of the effective dose for one scan were shown in Figure 3. A large variation was observed in the effective dose of one scan among institutions (CT units). In particular, in lower abdominal CT scans, the estimated effective dose ranged from 2.6 to $19.0 \mathrm{mSv}$. However, there were no factors which were significantly correlated with the estimated effective doses.

\section{- Estimated annual collective dose, and annual effective dose per capita in Japan}

The estimated annual collective effective dose in Japan was $277.4 * 10^{3} \mathrm{~Sv}$ person, and CT examinations of the chest and upper abdomen contributed to approximately
$73.2 \%$ of the collective dose (Table 3 ). The annual effective dose from CT examinations per capita in Japan was estimated at $2.20 \mathrm{mSv}$.

\section{Discussion}

The estimated annual number of the patients undergoing CT examinations in this study (235.4 per 1000 population) was slightly lower than that surveyed in 2000 (290 per 1000 population) [13]. Recent advances in CT technology, which enable us to obtain a wide range of CT images, such as neck through the lower abdomen in one session, may partially explain the decrease in the number of the patients undergoing CT examinations. The data of the previous study [13] was obtained from randomlyselected hospitals around Japan, and each hospital provided basic data for a single given week or a single given day. We collected data for a single month from the institutions in a single prefecture. The differences in data

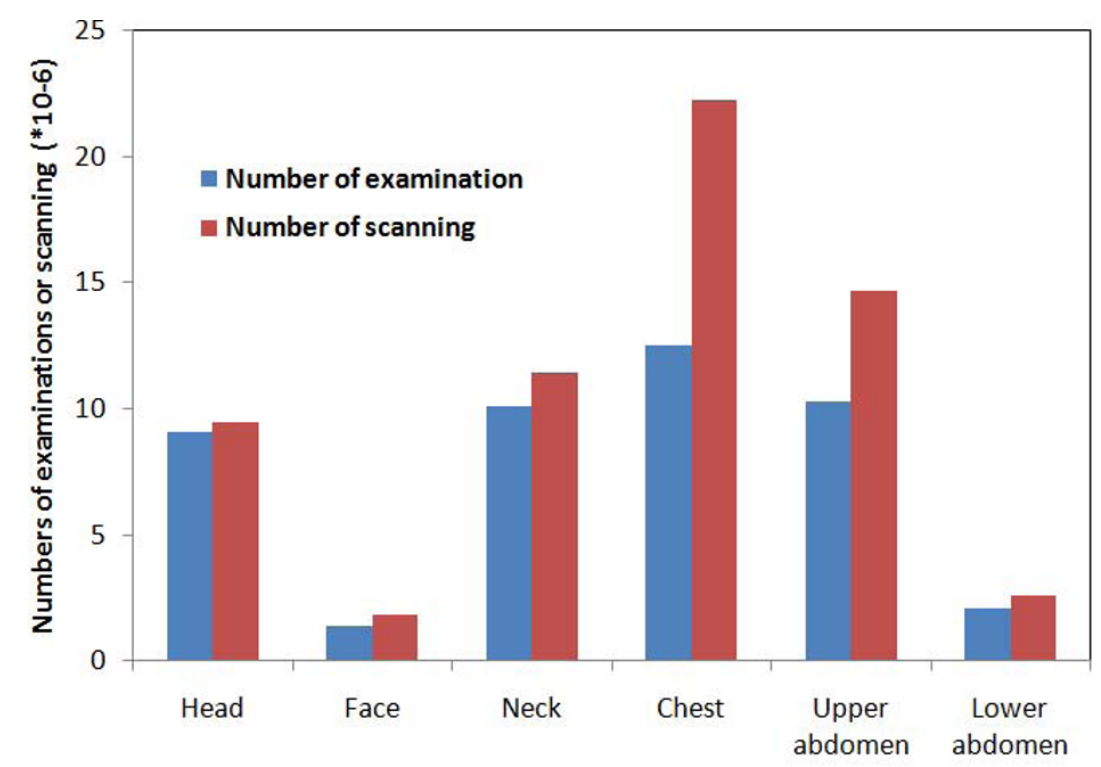

Anatomical location

Figure 2 Estimated annual numbers of CT examinations and scannings in Japan. The estimated annual number of patients who underwent CT in Japan was approximately 29.9 million. In approximately 50\% of these patients, more than two anatomical locations were scanned in one CT session, thus the number of CT examinations was about 45.3 million annually. In addition, approximately $37 \%$ of the patients underwent more than two scans of the same anatomical location (for instance, unenhanced and enhanced CT), making the number of scans about 62.5 million annually. 
Table 3 Estimated annual number of CT examinations and collective dose in Japan

\begin{tabular}{|c|c|c|c|}
\hline CT examination & & Annual number of CT examination $\left(* 10^{6}\right)$ & $\begin{array}{l}\text { Annual collective dose } \\
\left({ }^{*} 10^{3} \mathrm{~Sv} \text { person) }\right.\end{array}$ \\
\hline \multirow[t]{3}{*}{ Head } & Total & 9.1 & 23.6 \\
\hline & Men & 4.6 & 12.1 \\
\hline & Women & 4.5 & 11.5 \\
\hline \multirow[t]{3}{*}{ Face } & Total & 1.4 & 2.8 \\
\hline & Men & 0.7 & 1.5 \\
\hline & Women & 0.7 & 1.3 \\
\hline \multirow[t]{3}{*}{ Neck } & Total & 10.1 & 26.5 \\
\hline & Men & 5.6 & 14.8 \\
\hline & Women & 4.5 & 11.7 \\
\hline \multirow[t]{3}{*}{ Chest } & Total & 12.5 & 120.0 \\
\hline & Men & 7.0 & 67.8 \\
\hline & Women & 5.5 & 52.2 \\
\hline \multirow[t]{3}{*}{ Upper abdomen } & Total & 10.3 & 83.1 \\
\hline & Men & 5.7 & 47.1 \\
\hline & Women & 4.6 & 36.0 \\
\hline \multirow[t]{3}{*}{ Lower abdomen } & Total & 2.1 & 21.3 \\
\hline & Men & 1.1 & 12.0 \\
\hline & Women & 1.0 & 9.3 \\
\hline \multirow[t]{3}{*}{ Total } & Total & 45.4 & 277.4 \\
\hline & Men & 24.7 & 155.3 \\
\hline & Women & 20.8 & 122.1 \\
\hline
\end{tabular}

collection methods may be the reason for the slightly different results.

The collective effective dose $\left(277.4 * 10^{3} \mathrm{~Sv}\right.$ person $)$ estimated in the current study was slightly lower than that estimated in 2000 for Japan $\left(295 * 10^{3} \mathrm{~Sv}\right.$ person) [13]. This difference may be partially explained by the decrease in radiation exposure to the upper abdomen: the effective dose for one upper abdominal CT examination $(8.1 \mathrm{mSv})$ was $37 \%$ lower than that obtained in the previous Japanese study (12.9 mSv) (Table 6) [13], and CT examinations of the upper abdomen were frequent (23\%). The advance of multi-detector CT technology may have decreased the radiation dose [14]. In the previous Japanese study [13], the collective effective dose for head CTs was estimated around $38 * 10^{3}$ Sv person, which was much higher than our result $\left(23.6 * 10^{3} \mathrm{~Sv}\right.$ person $)$ in spite of an almost equal effective dose for a single examination ( 2.4 vs. $2.6 \mathrm{mSv}$ ). We suspect this decrease may be due to the recent shift from CT to MRI as the mainstay of radiologic examinations of the head.

Radiation dose also varied among countries [13-16]. Although the reasons for these variations are unclear for us, the average doses in the UK are generally lower not only than those of Japan, but also those of Germany and Netherlands.

- Uncertainties in the estimation, and limitations of this study

From this study, we learned there were significant difficulties in estimating the radiation exposure from CT

Table 4 Estimated annual number of CT examinations for men (a) and for women (b) in Japan for each age range and anatomical locations scanned $\left(* 10^{6}\right)$

\begin{tabular}{|c|c|c|c|c|c|c|c|c|c|}
\hline \multirow[b]{2}{*}{ CT examination } & \multicolumn{8}{|c|}{ Age range (yo) } & \multirow[b]{2}{*}{ Total } \\
\hline & $0-19$ & $20-29$ & $30-39$ & $40-49$ & $50-59$ & $60-69$ & $70-79$ & $80-$ & \\
\hline Head & 0.43 & 0.17 & 0.24 & 0.36 & 0.53 & 0.89 & 1.11 & 0.89 & 4.62 \\
\hline Face & 0.12 & 0.06 & 0.06 & 0.06 & 0.10 & 0.14 & 0.08 & 0.07 & 0.69 \\
\hline Neck & 0.17 & 0.10 & 0.28 & 0.36 & 0.86 & 1.66 & 1.53 & 0.64 & 5.60 \\
\hline Chest & 0.09 & 0.10 & 0.25 & 0.43 & 0.91 & 1.86 & 2.10 & 1.26 & 7.00 \\
\hline Upper abdomen & 0.10 & 0.09 & 0.21 & 0.36 & 0.74 & 1.58 & 1.67 & 0.94 & 5.69 \\
\hline Lower abdomen & 0.02 & 0.02 & 0.04 & 0.07 & 0.13 & 0.31 & 0.32 & 0.18 & 1.09 \\
\hline Total & 0.93 & 0.54 & 1.08 & 1.64 & 3.27 & 6.44 & 6.81 & 3.98 & 24.69 \\
\hline
\end{tabular}


Table 5 Estimated annual number of CT examinations for men (a) and for women (b) in Japan for each age range and anatomical locations scanned $\left(* 10^{6}\right)$

\begin{tabular}{lrrrrrrrrr}
\hline \multicolumn{1}{c}{ Age range (yo) } \\
\hline CT examination & $\mathbf{0 - 1 9}$ & $\mathbf{2 0 - 2 9}$ & $\mathbf{3 0 - 3 9}$ & $\mathbf{4 0 - 4 9}$ & $\mathbf{5 0 - 5 9}$ & $\mathbf{6 0 - 6 9}$ & $\mathbf{7 0 - 7 9}$ & $\mathbf{8 0 -}$ & Total \\
\hline Head & 0.10 & 0.17 & 0.23 & 0.29 & 0.50 & 0.73 & 1.14 & 1.34 & 4.50 \\
Face & 0.11 & 0.04 & 0.04 & 0.05 & 0.09 & 0.13 & 0.16 & 0.08 & 0.70 \\
Neck & 0.00 & 0.08 & 0.27 & 0.59 & 0.97 & 1.14 & 0.96 & 0.50 & 4.51 \\
Chest & 0.09 & 0.06 & 0.22 & 0.42 & 0.90 & 1.21 & 1.30 & 1.30 & 5.50 \\
Upper abdomen & 0.00 & 0.09 & 0.22 & 0.39 & 0.76 & 1.03 & 1.16 & 0.94 & 4.59 \\
Lower abdomen & 0.00 & 0.03 & 0.05 & 0.09 & 0.16 & 0.21 & 0.24 & 0.22 & 1.00 \\
\hline Total & 0.30 & 0.47 & 1.03 & 1.83 & 3.38 & 4.45 & 4.96 & 4.38 & 20.80 \\
\hline
\end{tabular}

examinations. There is a large uncertainty associated with the radiation dose estimates in this study. We would like to discuss the uncertainty in the estimation, and limitations of this study. There are two sources of the uncertainties in the estimation in this study: organ dose estimates, and the sample of patients studied.

The study questionnaire surveyed only the standard CT protocols for each anatomical location, but radiologic technologists usually modify the protocol according to patient body size, affecting the radiation dose. Some CT examinations may be performed for screening purposes, and the screening protocol used in these cases may lead to lower dose values than the standard protocol. In the current study, this possibility was not considered, although screening CT has yet to become popular in Japan.

We collected basic data from hospitals and clinics in a single prefecture to estimate the overall situation in Japan. The age distribution of Gunma prefecture is very similar to that of Japan as a whole, and we suspect that the medical environment in Gunma prefecture reflects that of the Japanese national average (Figure 1; Table 1). However, factors such as economic status and access to hospital care may influence medical care a population receives, including medical radiation exposure. Since the average annual medical cost per population was slightly lower in Gunma prefecture than the national average [17-21], basing our estimates on the number of patients undergoing CT in Gunma prefecture may potentially underestimate the number of patients undergoing $\mathrm{CT}$ in Japan as a whole by approximately $10 \%$.

In this study, the data was obtained from half of the hospitals and clinics to which the survey was sent. We are not sure whether or not the radiology departments which provided information differ in numbers and types of CT examinations from those which did not provide information. The data was obtained during June, and multiplied by 12 to obtain the annual estimates. June may not be a typical month regarding $\mathrm{CT}$ examinations,

Table 6 Estimated effective doses per scan and examination for each anatomical location in Japan, and comparison with previous studies

\begin{tabular}{|c|c|c|c|c|c|c|}
\hline \multirow[t]{2}{*}{ CT examination } & \multicolumn{5}{|c|}{ Effective dose for one scanning/one examination (mSv) } & \multirow[b]{2}{*}{$\begin{array}{l}2003-2004 \\
\text { Netherlands (14) }\end{array}$} \\
\hline & $\begin{array}{l}\text { Year } \\
\text { Study }\end{array}$ & $\begin{array}{l}2008 \\
\text { Current (Japan) }\end{array}$ & $\begin{array}{l}2000 \\
\text { Japan (13) }\end{array}$ & $\begin{array}{l}2002 \\
\text { Germany (15) }\end{array}$ & $\begin{array}{l}2003 \\
\text { UK (16) }\end{array}$ & \\
\hline \multirow[t]{2}{*}{ Head } & Scan & 2.5 & - & 2.2 & $0.8^{*}$ & 1.9 \\
\hline & Examination & 2.6 & 2.4 & 2.8 & 1.5 & - \\
\hline \multirow[t]{2}{*}{ Face } & Scan & 1.5 & - & 0.8 & - & - \\
\hline & Examination & 2.0 & - & 0.8 & - & - \\
\hline \multirow[t]{2}{*}{ Neck } & Scan & 2.3 & - & - & - & - \\
\hline & Examination & 2.6 & - & - & - & - \\
\hline \multirow[t]{2}{*}{ Chest } & Scan & 5.4 & - & 5.5 & $3.4^{*}$ & 3.8 \\
\hline & Examination & 9.6 & 9.1 & 5.7 & 5.8 & - \\
\hline \multirow[t]{2}{*}{ Upper abdomen } & Scan & 5.7 & - & 5.5 & $3.8^{*}$ & 7.2 \\
\hline & Examination & 8.1 & 12.9 & 11.5 & 5.3 & $10.2^{* *}$ \\
\hline \multirow[t]{2}{*}{ Lower abdomen } & Scan & 8.3 & - & 6.3 & - & - \\
\hline & Examination & 10.3 & 10.5 & 7.2 & - & - \\
\hline
\end{tabular}

-: no data.

*: Effective doses for one examination were divided by the number of scanning of the routine sequences.

**: Sum of the effective doses of arterial and portal scans. 


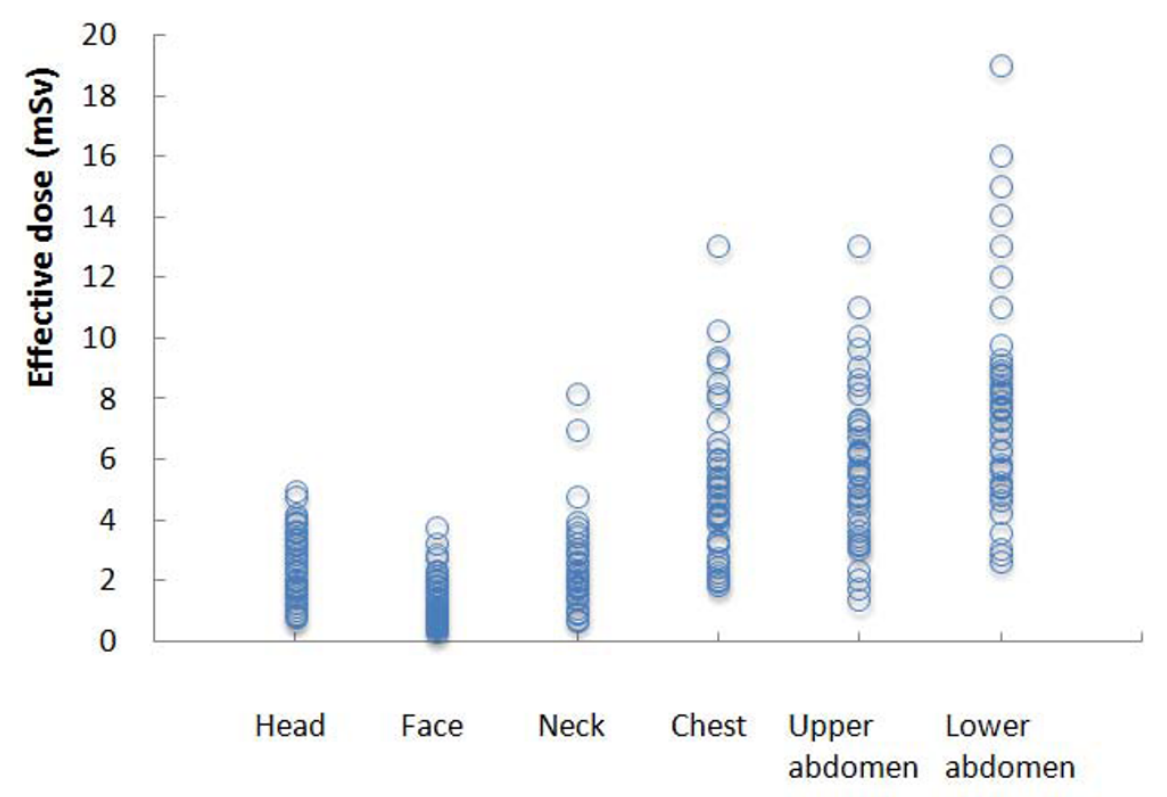

\section{Anatomical location}

Figure 3 Distribution of effective dose for one scan for each anatomical location in Gunma prefecture. The radiation dose of CT examinations varies greatly among institutions.

but the differences in the number of CT examinations throughout the calendar year in Japan were reported to be less than $2 \%[13]$.

Paediatric patients were not included in radiation exposure estimates, since calculating paediatric exposure is problematic. Because of the higher radiation doses to paediatric patients for a given CT $[7,22]$, we suspect the issue of medical radiation exposure to the paediatric patients should be separately discussed. Cardiac CT, which has been recently introduced to clinical practice, was also excluded from our analysis. It has been reported that organ doses to lungs and breast are relatively large in cardiac CT $[2,8]$. Although the individual radiation exposure is relatively large, we suspect this may not greatly affect the overall results of this study.

Despite these limitations, we believe that the simple approach used in this study to estimate radiation exposure from CT examinations is the best available from current data and technology.

\section{- A large variation in estimated dose among CT scanners, and the methods reducing radiation exposure}

There was a large variation in estimated dose among CT scanners. In particular, for lower abdominal $\mathrm{CT}$, the radiation exposure ranged from 2.6 to $19 \mathrm{mSv}$ (Figure 3 ). This finding indicated that in some institutions, patients probably receive unnecessary radiation exposure, but in others the radiation exposure may not be sufficient to obtain adequate image quality. However, we have failed to find factors which may contribute to the large variation of radiation exposure. In Japan, there is no official system responsible for quality control of CT examinations and radiation exposure to patients, in spite of very strict governmental control of radioisotope management and radiation exposure to medical professionals. We would like to emphasize the importance of a quality control system for CT image quality and patient radiation exposure. Proper feedback, increased education and application of reference dose levels will be important tools to further reduce such institutional differences $[9,14]$.

Some reliable methods which significantly reduce radiation exposure to patients from $\mathrm{CT}$ have been proposed [10]. AEC systems adjust radiation dose according to the patient's attenuation, and may reduce the mean tube current by $20-68 \%$ (for instance, $20 \%$ for thorax, $38 \%$ for abdomen, and $32 \%$ for abdomen-pelvis) $[11,23,24]$. This system is installed in recent modern CT scanners, and usually sustains image quality without an increase of noise. In this study, less than half of the CT scanners surveyed had AEC system.

Of course, the most effective way to reduce the radiation exposure to the population from $\mathrm{CT}$ is to avoid unnecessary CT examinations. There is a recent trend for diagnostic imaging to be performed as a precaution to avoid malpractice suits (defensive medicine) $[25,26]$. In addition, we suspect a tendency for Japanese clinicians to rely heavily on imaging examinations because 
of their extremely heavy workload due to the recent critical shortage of physicians. Patients also seek perfect medicine, and request imaging examinations themselves, which are provided at a relatively modest price in Japan $[26,27]$. Physicians should carefully consider the risks and benefits before ordering CT.

\section{Conclusions}

The annual number of adult patients undergoing CT examinations in Japan was estimated at 29.9 million, and that of paediatric patients at 1.43 million. The estimated annual collective effective dose in Japan was $277.4 * 10^{3} \mathrm{~Sv}$ person. The CT examinations of the chest and upper abdomen predominantly contribute to $73.2 \%$ of the collective dose. There was a very large variation in radiation exposure from $\mathrm{CT}$ among hospitals surveyed.

\section{Acknowledgements}

This work was supported in part by the Grant-in-Aid for Cancer Research [19-22] from the Ministry of Health, Labour and Welfare, Japan.

\section{Authors' contributions}

Conception and design: YT \& KE.

Acquisition of data and analysis: YT, HT, HO \& KE.

Interpretation of data: YT, ATT \& KE.

All authors read and approved the final manuscript.

\section{Competing interests}

The authors declare that they have no competing interests.

Received: 30 March 2010 Accepted: 2 November 2010

Published: 2 November 2010

\section{References}

1. Berrington De Gonzalex A, Darby S: Risk of cancer from diagnostic X-rays: estimates for the UK and 14 other countries. Lancet 2004, 363:345-51.

2. Einstein AJ, Henzlova MJ, Rajagopalan S: Estimating risk of cancer associated with radiation exposure from 64-slice computed tomography coronary angiography. JAMA 2007, 298:317-323.

3. Hall EJ, Brenner DJ: Cancer risks from diagnostic radiology. $\mathrm{Br} J$ Radiol 2008, 81:362-378.

4. Brix G, Nissen-Meyer S, Lechel U, et al: Radiation exposures of cancer patients from medical X-rays: How relevant are they for individual patients and population exposure? Eur J Radiol 2009, 72:342-347.

5. Mettler FA Jr, Wiest PW, Locken JA, Kelsey CA: CT scanning: patterns of use and dose. J Radiol Prot 2000, 20:353-359.

6. Ministry of Internal Affairs and communications: Result of the Population Estimates 2007 [http://www.e-stat.go.jp/SG1/estat/List.do? bid=000001007609\&cycode=0], (in Japanese).

7. Brenner DJ, Hall EJ: Computed tomography: An increasing source of radiation exposure. N Engl J Med 2007, 357:2277-2284.

8. Hurwitz LM, Reiman RE, Yoshizumi $\Pi$, et al: Radiation dose from contemporary cardiothoracic multidetector CT protocols with an anthropomorphic female phantom: implication for cancer induction. Radiology 2007, 245:742-750.

9. Cho PK, Seo BK, Choi TK, Kim JS, Kim YH, Choi JH: The development of a diagnostic reference level on patient dose for $\mathrm{CT}$ examination in Korea. Radiat Prot Dosimetry 2008, 129:463-8.

10. Kalra MK, Maher MM, Toth $T L$, et al: Strategies for CT radiation dose optimization. Radiology 2004, 230:619-628.

11. Greess $H$, Wolf $H$, Baum $U$, et al: Dose reduction in computed tomography by attenuation-based on-line modulation of tube current: evaluation of six anatomical regions. Eur Radiol 2000, 10:391-394.

12. Jones DG, Shrimpton PC: NRPB-SR250: Normalised Organ Doses for X-Ray Computed Tomography Calculated Using Monte Carlo Techniques 2008
[http://www.hpa.org.uk/web/HPAweb\&HPAwebStandard/HPAweb_C/ $1195733753330]$.

13. Nishizawa K, Matsumoto M, Iwai K, Maruyama T: Survey of CT practice in Japan and collective effective dose estimation. Nippon Acta Radiol 2004, 64:151-158.

14. Van der Molen AJ, Veldkamp WJH, Geleijns J: 16-slice CT: achievable effective doses of common protocols in comparison with recent CT dose surveys. Br J Radiol 2007, 80:248-255.

15. Brix G, Nagel HD, Stamm G, Veit R, Lechel U, Griebel J, Galanski M: Radiation exposure in multi-slice versus single-slice spiral $\mathrm{CT}$ : results of a nationwide survey. Eur Radiol 2003, 13:1979-1991.

16. Shrimpton PC, Hillier MC, Lewis MA, Dunn M: Doses from computed tomography examinations in the UK - 2003 review. Report NRPB-W67 2005 [http://www.hpa.org.uk/Publications/Radiation/NPRBArchive/ NRPBWSeriesReports/2005nrpbw067/].

17. Ministry of Health, Labour and Welfare: Numbers of physicians, dentists and pharmacists per 100,000 populations 2006 [http://www.mhlw.go.jp/toukei/ saikin/hw/ishi/06/tou13.html].

18. Ministry of Health, Labour and Welfare: Annual medical costs per populations 2005 [http://www.mhlw.go.jp/topics/bukyoku/hoken/iryomap/05/01.html].

19. Ministry of Health, Labour and Welfare: Numbers of hospitals per populations 2007 [http://www.mhlw.go.jp/toukei/saikin/hw/iryosd/07/toukei08.html].

20. Ministry of Health, Labour and Welfare: Numbers of beds per populations 2007 [http://www.mhlw.go.jp/toukei/saikin/hw/iryosd/07/toukei09.html].

21. Ministry of Internal Affairs and communications: Basic economics 2004 [http://www.stat.go.jp/index.html]

22. Brenner DJ, Elliston CD, Hall EJ, Berdon WE: Estimated risks of radiationinduced fatal cancer from pediatric CT. AJR 2001, 176:289-296.

23. Lee $\mathrm{CH}, \mathrm{Goo} \mathrm{JM}, \mathrm{Ye} \mathrm{HJ}$, et al: Radiation dose modulation techniques in the multidetector CT era: From basics to practice. RadioGraphics 2008, 28:1451-1459.

24. Mulkens $\mathrm{TH}$, Bellinck $P$, Baeyaert $M$, et al: Use of an automatic exposure control mechanism for dose optimization in multi-detector row CT examinations: Clinical evaluation. Radiology 2005, 237:213-223.

25. Berlin L: Errors of omission. AJR 2005, 185:1416-1421.

26. Nakajima Y, Yamada K, Imamura K, Kobayashi K: Radiologist supply and workload: international comparison-Working group of Japanese college of radiology. Radiat Med 2008, 26:455-465.

27. Katada K: CT radiation exposure in Japan: analysis of social factors. Jpn J Radiol Technol 2006, 62:649-656.

Pre-publication history

The pre-publication history for this paper can be accessed here: http://www.biomedcentral.com/1471-2342/10/24/prepub

\section{doi:10.1186/1471-2342-10-24}

Cite this article as: Tsushima et al:: Radiation Exposure from CT Examinations in Japan. BMC Medical Imaging 2010 10:24.

\section{Submit your next manuscript to BioMed Central and take full advantage of:}

- Convenient online submission

- Thorough peer review

- No space constraints or color figure charges

- Immediate publication on acceptance

- Inclusion in PubMed, CAS, Scopus and Google Scholar

- Research which is freely available for redistribution 